(1)

CrossMark

\title{
Pharmacological management of progressive-fibrosing interstitial lung diseases: a review of the current evidence
}

\author{
Luca Richeldi ${ }^{1}$, Francesco Varone ${ }^{1}$, Miguel Bergna ${ }^{2}$, Joao de Andrade ${ }^{3}$, \\ Jeremy Falk ${ }^{4}$, Robert Hallowell ${ }^{5}$, Stéphane Jouneau ${ }^{6}$, Yasuhiro Kondoh ${ }^{7}$, \\ Lee Morrow ${ }^{8}$, Winfried Randerath ${ }^{9}$, Mary Strek ${ }^{10}$ and Gabriela Tabaj ${ }^{11}$
}

Affiliations: ${ }^{1}$ Unità Operativa Complessa di Pneumologia, Fondazione Policlinico Universitario A. Gemelli IRCSS, Università Cattolica del Sacro Cuore, Rome, Italy. ${ }^{2}$ Centro Médico de Enfermedades, Respiratorias, Florida, Vicente López, Buenos Aires, Argentina. ${ }^{3}$ University of Alabama at Birmingham, Birmingham, $A L$, USA. ${ }^{4}$ Cedars-Sinai Medical Center, Division of Pulmonary and Critical Care Medicine, Los Angeles, CA, USA. ${ }^{5}$ Division of Pulmonary, Critical Care, and Sleep Medicine, Beth Israel Deaconess Medical Center, Harvard Medical School, Boston, MA, USA. ${ }^{6}$ Respiratory Diseases Dept, Hôpital Pontchaillou, IRSET, Université de Rennes 1, Rennes, France. ${ }^{7}$ Dept of Respiratory Medicine and Allergy, Tosei General Hospital, Seto, Japan. ${ }^{8}$ Division of Pulmonary, Critical Care and Sleep Medicine, Dept of Internal Medicine, Veterans Affairs Nebraska-Western lowa Health Care System, Omaha, NE, USA. ${ }^{9}$ Institute of Pneumology, University of Cologne, Bethanien Hospital, Solingen, Germany. ${ }^{10}$ Dept of Radiology, University of Chicago, Chicago, IL, USA. ${ }^{11}$ Pulmonary Medicine, Cetrángolo Hospital, Buenos Aires, Argentina.

Correspondence: Luca Richeldi, Unità Operativa Complessa di Pneumologia, Fondazione Policlinico Universitario A. Gemelli IRCSS, Università Cattolica del Sacro Cuore, Largo Gemelli, 00168, Rome, Italy. E-mail: luca.richeldiđpoliclinicogemelli.it

@ERSpublications

Aside from IPF, there are no proven therapies for other ILDs with a progressive-fibrosing phenotype that are effective and have tolerable adverse effects. Clinical studies evaluating the benefits of antifibrotic therapy in these populations are underway. http://ow.ly/40yL30mOs0q

Cite this article as: Richeldi L, Varone F, Bergna M, et al. Pharmacological management of progressivefibrosing interstitial lung diseases: a review of the current evidence. Eur Respir Rev 2018; 27: 180074 [https://doi.org/10.1183/16000617.0074-2018].

ABSTRACT A proportion of patients with interstitial lung diseases (ILDs) are at risk of developing a progressive-fibrosing phenotype, which is associated with a deterioration in lung function and early mortality. In addition to idiopathic pulmonary fibrosis (IPF), fibrosing ILDs that may present a progressive phenotype include idiopathic nonspecific interstitial pneumonia, connective tissue disease-associated ILDs, hypersensitivity pneumonitis, unclassifiable idiopathic interstitial pneumonia, ILDs related to other occupational exposures and sarcoidosis. Corticosteroids and/or immunosuppressive therapies are sometimes prescribed to patients with these diseases. However, this treatment regimen may not be effective, adequate on its own or well tolerated, suggesting that there is a pressing need for efficacious and better tolerated therapies. Currently, the only approved treatments to slow disease progression in patients with IPF are nintedanib and pirfenidone. Similarities in pathobiological mechanisms leading to fibrosis between IPF and other ILDs that may present a progressive-fibrosing phenotype provide a rationale to suggest that nintedanib and pirfenidone may be therapeutic options for patients with the latter diseases.

This review provides an overview of the therapeutic options currently available for patients with fibrosing ILDs, including fibrosing ILDs that may present a progressive phenotype, and explores the status of the randomised controlled trials that are underway to determine the efficacy and safety of nintedanib and pirfenidone.

Copyright OERS 2018. ERR articles are open access and distributed under the terms of the Creative Commons Attribution Non-Commercial Licence 4.0. 


\section{Introduction}

Interstitial lung diseases (ILDs) are a group of rare respiratory, nonmalignant disorders, characterised by varying degrees of damage to the lung parenchyma via inflammation and fibrosis $[1,2]$. While idiopathic pulmonary fibrosis (IPF) may be regarded as the prototype progressive-fibrosing ILD [3], a proportion of patients with other ILDs develop a similar progressive-fibrosing phenotype. The pathophysiology in these patients is characterised by self-sustaining fibrosis and a deterioration in lung function over time, with worsening respiratory symptoms, resistance to immunomodulatory therapies and ultimately early mortality [1]. Terminology recently used to describe patients with fibrosing ILDs that may present a progressive phenotype is "progressive-fibrosing ILD" (PF-ILD) [1]. For more information refer to the article by CoTTIN et al. [4] in this issue of the European Respiratory Review. Treatment of these fibrotic lung diseases is associated with a number of challenges including, but not limited to: difficulties in classification and/or differential diagnosis, variability in the clinical course of the disease, and a need for biomarkers to help predict disease progression [5]. Immunosuppressive agents are sometimes used off-label in patients with ILDs, but they may potentially cause interstitial changes that worsen the disease. While corticosteroids with or without immunosuppressive drugs were historically chosen as first-line treatment, they may not be effective. Therefore, except for nintedanib and pirfenidone, which have been approved specifically for the treatment of IPF, there is currently a lack of safe and effective pharmacological treatments to slow disease progression in ILDs that may present a progressive-fibrosing phenotype [6-8].

\section{Management of IPF}

IPF is a fatal disease, its clinical course in the individual patient is variable and, therefore, unpredictable $[9,10]$. The current aims of patient care are to improve outcomes by slowing disease progression and extending and improving quality of life (QoL) [11]. Antifibrotic treatments are usually prescribed with the aim to slow down disease progression [12]. The most recent international evidence-based guidelines issued by the American Thoracic Society, the European Respiratory Society, the Japanese Respiratory Society and the Latin American Thoracic Association provide conditional recommendations for the use of two antifibrotic treatments, nintedanib and pirfenidone, which have been shown to reduce the decline in lung function in patients with IPF $[6,11,13-15]$. The same guidelines provide strong recommendations against the use of ambrisentan, anticoagulants, the combination of prednisone, azathioprine and $\mathrm{N}$-acetylcysteine (NAC) and imatinib $[6,11]$. The guidelines also make conditional recommendations against the use of dual endothelial receptor antagonists, NAC monotherapy and sildenafil $[6,11]$.

\section{Nintedanib}

Nintedanib is a small, potent, oral intracellular tyrosine kinase inhibitor, which inhibits downstream signalling pathways involved in fibrogenesis [16-18]. Nintedanib competitively binds to the adenosine triphosphate binding pocket of these receptors inhibiting the activation of the platelet-derived growth factor receptor, vascular endothelial growth factor receptor and fibroblast growth factor receptors signalling cascades, which are critically involved in the proliferation, migration and differentiation of lung fibroblasts/myofibroblasts, the hallmark cells in the pathology of IPF [7, 16-18]. In addition, nintedanib has also been shown to inhibit nonreceptor tyrosine kinases of the Src family including Src, Lck and Lyn [16, 17]. Lck inhibition may reduce downstream signalling of human T-cell receptors to reduce the release of immune-stimulating and profibrotic mediators such as interferon- $\gamma$ and several other interleukins [19]. This inhibition may be particularly important in the treatment of connective tissue disease-associated ILDs (CTD-ILDs) [19, 20].

\section{Approval of nintedanib}

In 2014, nintedanib was approved for the treatment of IPF in the USA by the Food and Drug Administration, and in Japan and Europe in 2015 [7, 21, 22]. The conditional recommendation for the use of nintedanib in international guidelines was based on the outcomes from three clinical studies, TOMORROW, INPULSIS-1 and INPULSIS-2 $[6,13,14]$. The phase II TOMORROW trial showed that nintedanib, $150 \mathrm{mg}$ twice daily, effectively reduced the rate of decline in forced vital capacity (FVC) by $68.4 \%$ versus placebo $(60 \mathrm{~mL}$ and $190 \mathrm{~mL}$, respectively; $\mathrm{p}=0.01$ ) (figure 1a) [12]. Outcomes from INPULSIS- 1 and INPULSIS-2 trials showed that the annual rate of decline in FVC was significantly reduced in the nintedanib group compared with placebo (INPULSIS-1: $125.3 \mathrm{~mL} \cdot \mathrm{year}^{-1}\left(-114.7 \mathrm{~mL} \cdot \mathrm{year}^{-1}\right.$

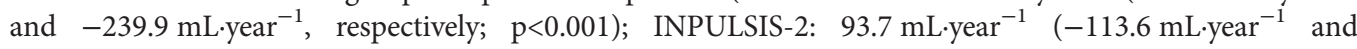

Provenance: Publication of this peer-reviewed article was sponsored by Boehringer Ingelheim Pharmaceuticals Inc, Ridgefield, CT, USA (principal sponsor, European Respiratory Review issue 150).

Received: Aug 142018 | Accepted after revision: Oct 302018

Published online Dec 21, 2018; republished Jan 04, 2019 with a correction to the colour key in figure 1. 

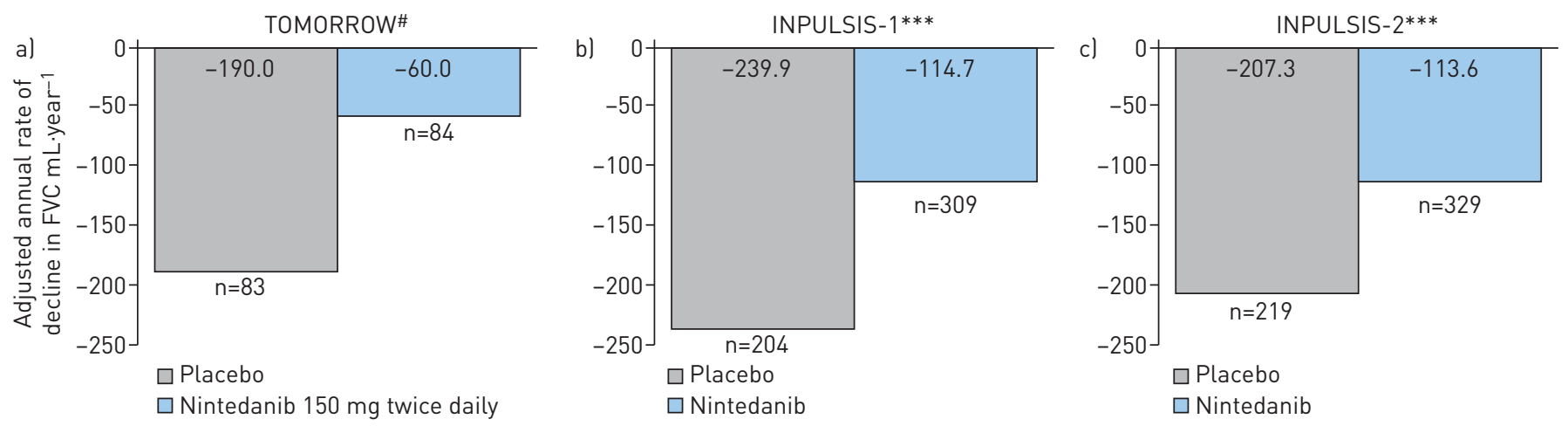

FIGURE 1 Annual rate of change from baseline over time in forced vital capacity (FVC) in the a) phase II TOMORROW [12], b) phase III INPULSIS-1 [13] and c) INPULSIS-2 [13] studies. ${ }^{\#}: p=0.01 ; * * *: p<0.001$.

$-207.3 \mathrm{~mL} \cdot \mathrm{year}^{-1}$, respectively; $\mathrm{p}<0.001$ )) (figure $1 \mathrm{~b}$ and c) [13]. All trials showed that nintedanib had a reasonable safety profile, with gastrointestinal events, particularly diarrhoea, being the most commonly reported adverse events (AEs).

A pooled and meta-analyses of the TOMORROW and INPULSIS studies demonstrated that nintedanib $150 \mathrm{mg}$ twice daily consistently slowed disease progression and was also associated with a reduction in the risk of acute IPF exacerbations, maintenance of health-related QoL and reduction in the risk of all-cause mortality [19]. Diarrhoea was the most commonly reported AE in the nintedanib treatment group [10]. Based on the outcomes of these clinical studies, the recommended daily dose of nintedanib for patients with IPF is $150 \mathrm{mg}$ twice daily, administered every $12 \mathrm{~h}$, with the option to reduce the dose to $100 \mathrm{mg}$ twice daily to manage side-effects [7].

\section{Pirfenidone}

Pirfenidone is an oral agent that has been shown to have anti-inflammatory, antioxidative and antiproliferative properties in a number of cellular and animal models of inflammation and fibrosis [23]. In several models of inflammation, pirfenidone has been shown to reduce secreted levels of bioactive and cell-associated tumour necrosis factor (TNF)- $\alpha$ after stimulation with a lipopolysaccharide [23]. However, how pirfenidone exerts its antifibrotic and anti-inflammatory properties has not yet been fully established [23].

\section{Approval of pirfenidone}

Pirfenidone was approved in Japan in 2008 (Pirespa; Shionogi \& Co., Ltd, Japan) [24] for the treatment of IPF, in Europe in 2011 (Esbriet; Roche Registration GmbH, Switzerland) for patients with mild-to-moderate IPF and in the USA in 2014 (Esbriet; Roche, USA) [25] for patients with IPF [24, 26]. The conditional recommendation in international guidelines for the use of pirfenidone in patients with IPF was based on the outcomes of three phase III clinical studies, CAPACITY-004, CAPACITY-006 and ASCEND [6, 15, 27].

Results from the two concurrent phase III studies (CAPACITY-004 and CAPACITY-006) showed that the mean decline in FVC \% predicted at 72 weeks of treatment was significantly reduced with pirfenidone versus placebo (CAPACITY-004: $-8.0 \%$ versus -12.4 ; $\mathrm{p}=0.001$; figure $2 \mathrm{a}$ ) and the decline in FVC \% pred
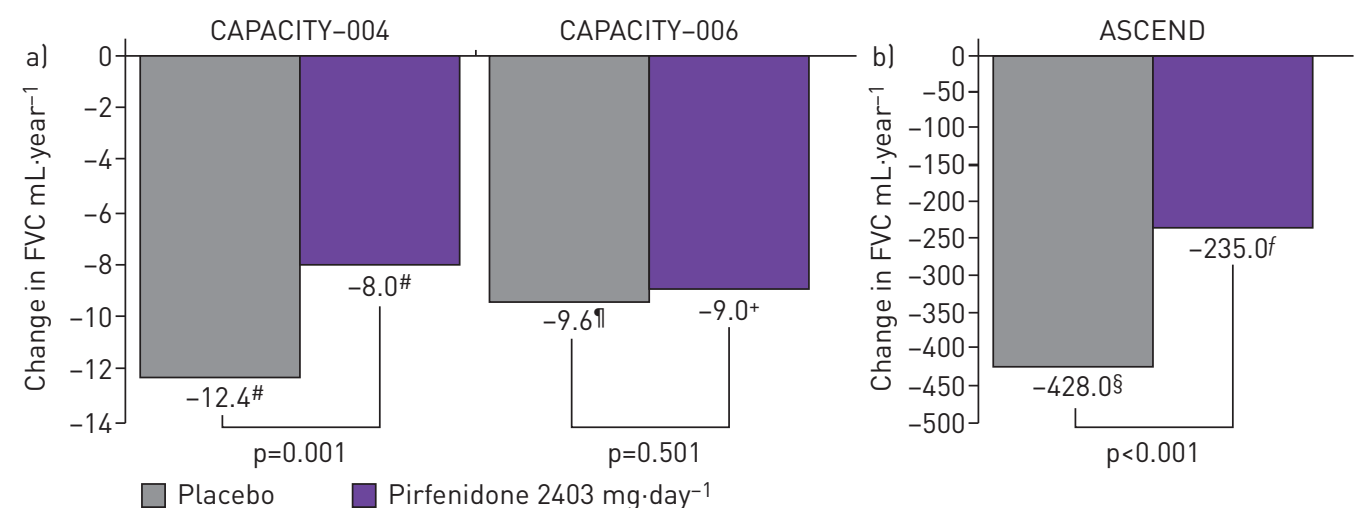

FIGURE 2 Mean change from baseline in percentage predicted forced vital capacity (FVC) in the a) phase III CAPACITY [27] and b) ASCEND [14] studies. ${ }^{\#}: n=174 ;{ }^{n}: n=173^{+}: n=171 ;{ }^{\S}: n=277 ;{ }^{f}: n=278$. Reproduced from [14] with permission. 
was $-9.0 \%$ ( pirfenidone) versus $-9.6 \%$ (placebo) (CAPACITY-006: $\mathrm{p}=0.501$; figure 2b) [27]. Pirfenidone was generally well-tolerated, and no significant treatment group differences were reported in either study in terms of acute exacerbations (worsening IPF) [27]. In the ASCEND study, at week 52, the proportion of patients who experienced a decline of 10 percentage points or more in predicted FVC, or who died, was reduced by $47.9 \%$ in the pirfenidone group compared with the placebo group $(16.5 \%$ and $31.8 \%$, respectively) (figure 2b) [15]. Gastrointestinal and skin-related events were more common in the pirfenidone group than the placebo group [15].

Based on findings from these trials, the recommended daily dose of pirfenidone in patients with IPF was three $267 \mathrm{mg}$ capsules three times daily with food for a total of $2403 \mathrm{mg} \cdot \mathrm{day}^{-1}$ [8]. The dose should be titrated to the recommended daily dose of nine capsules per day over a 14-day period as follows: days 1-7: one capsule three times daily $\left(801 \mathrm{mg} \cdot \mathrm{day}^{-1}\right)$; days $8-14$ : two capsules, three times daily $\left(1602 \mathrm{mg}^{- \text {day }^{-1}}\right)$; day 15 onward: three capsules, three times daily $\left(2403 \mathrm{mg}^{- \text {day }^{-1}}\right.$ ) [8]. However, the dosing of pirfenidone in Japan is $1800 \mathrm{mg} \cdot \mathrm{day}^{-1}$ lower than in other regions, to account for differences in predicted bodyweights [28].

\section{Management of other ILDs at risk of a progressive-fibrosing phenotype Idiopathic nonspecific interstitial pneumonia}

The prognosis for patients with idiopathic nonspecific interstitial pneumonia (iNSIP) is variable [29, 30], with 5 -year survival rates being $\sim 82.3 \%[31,32]$. Patients with the cellular subtype of the disease generally respond to treatment with corticosteroids and immunosuppressive agents, and their prognosis is better than that of patients with IPF [33]. However, patients with the fibrotic subtype (prominent reticular changes and traction bronchiectasis according to high-resolution computed tomography (HRCT), evidence of fibrosis on biopsy, and a lack of lymphocytosis on bronchoalveolar lavage (BAL)) usually fare worse than those with cellular iNSIP (prominent lymphocytic inflammation evident from BAL and a mixed NSIP/organising pneumonia pattern) [34]. A number of studies have demonstrated the beneficial effects of corticosteroid therapy to help stabilise disease in patients with iNSIP. The Korean Interstitial Lung Disease Study Group surveyed patients with iNSIP treated with or without corticosteroids from 2003 to 2007 to compare the treatment response group (FVC improvement of $\geqslant 10 \%$ after 1 year) with the nonresponse group (FVC improvement <10\%) [35]. Corticosteroid treatment was associated with significant improvements in lung function over 1 year (FVC 10\%; forced expiratory volume (FEV1) 9.8\%; diffusing capacity of the lung for carbon monoxide $(D \mathrm{LCO}) 8.4 \%$; $<<0.001$ ) suggesting that early treatment should be considered in this patient population [35]. Immune-modulating drugs such as prednisone, azathioprine, cyclophosphamide and mycophenolate have been used empirically; however, their doses are based on clinical response as assessed by clinicians rather than evidence from randomised clinical trials [29, 36].

\section{Unclassifiable idiopathic interstitial pneumonia}

Between $10 \%$ and $38 \%$ of patients are believed to have unclassifiable idiopathic interstitial pneumonia [37, 38], which is unclassifiable, despite extensive clinical, radiological and/or pathological examination [5, 39]. Treatment for this patient population is based on the most probable diagnosis after detailed clinic radiological-pathological case discussions, and considerations of the expected disease behaviour [5, 39].

\section{CTD-ILDs}

Rheumatoid arthritis-ILD

The management of patients with rheumatoid arthritis associated-ILD (RA-ILD) continues to be a challenge, particularly as there are no international guidelines on the management of this specific disease manifestation [40]. A number of therapeutic agents such as corticosteroids in combination with other immunosuppressive agents have been suggested as being effective, but no large randomised controlled trials have been undertaken to guide clinical management of this disease complication [40-42]. Treatment options for RA-ILD are further complicated by commonly prescribed drugs of proven articular benefit including methotrexate, leflunomide and anti-TNF- $\alpha$ agents, being implicated in ex novo occurrence and acceleration of existing ILDs [41]. Corticosteroids in combination with immunosuppressive agents such as cyclosporine and cyclophosphamide may help with management of this disease; however, their efficacy in RA-ILD is usually extrapolated from experience with other CTD-ILDs due to a lack of evidence from controlled studies [41]. Rituximab and abatacept have been suggested as acceptable therapeutic choices for patients with RA-ILD; however, randomised studies are warranted to confirm these findings [43, 44]. Pirfenidone, which has proven efficacy in patients with IPF, is currently under investigation as a therapeutic option for patients with RA-ILD (ClinicalTrials.gov identifier: NCT02808871) [40, 45]. 
in randomised controlled trials, the outcomes of which resulted in the European League Against Rheumatism Scleroderma Trials and Research (EUSTAR) group recommending cyclophosphamide for the treatment of SSc-ILD [46]. The Scleroderma Lung Study I was the first multicentre, double-blind, placebo-controlled randomised trial evaluating the efficacy and safety of cyclophosphamide in patients with symptomatic SSc-ILD to demonstrate that cyclophosphamide improved lung function over a 1-year treatment period; however, a greater number of AEs were associated with cyclophosphamide than placebo [47]. Outcomes from a second year of follow-up suggested that the beneficial effects of 1-year of cyclophosphamide treatment on pulmonary function were no longer apparent at 24 months [48]. The benefits of cyclophosphamide in managing patients with SSc-ILD were also shown in a long-term, prospective, controlled study of patients with SSc-ILD randomised to receive cyclophosphamide or cyclophosphamide plus prednisone for 12 months [49]. After 1 year of treatment, FVC \% pred was comparable between both treatment groups. Interestingly in this study, 3 years after the end of treatment the FVC \% pred values were similar to those observed at 1 year, suggesting that cyclophosphamide may effectively stabilise lung function for longer than 12 months [49].

One safer, less toxic, alternative to cyclophosphamide for the management of several immune-mediated conditions is mycophenolate mofetil (MMF), with uncontrolled, prospective or retrospective case series suggesting that it may be effective in stabilising or improving lung function in these patients $[46,50,51]$. In particular, the Scleroderma II study, which was a large, double-blind, randomised, parallel-group study designed to evaluate the efficacy and safety of MMF compared with cyclophosphamide, which showed that both treatments were associated with significant improvements in lung function over 24 months [52]. However, as with the Scleroderma Lung Study I, cyclophosphamide was associated with more AEs [52].

Rituximab, a monoclonal antibody directed against the B-cell CD20 antigen is currently under investigation as a potential treatment option for SSc-ILD, with a number of small, nonrandomised studies demonstrating its efficacy in these patients [46]. A small study of eight patients receiving rituximab reported statistically significant improvements in FVC, DLCO and stabilisation of HRCT chest imaging findings compared with a matched control group [53]. Outcomes from a EUSTAR nested case-controlled study, in which nine patients had SSc-ILD, showed stable FVC and improved DLCO compared with baseline at follow-up in patients treated with rituximab $[46,54]$. Similar outcomes were reported in a small retrospective analysis of patients with SSc-ILD receiving rituximab who had failed other immunomodulatory treatments [55]. Recognising both the potential for rituximab to favourably impact lung function over the longer term [56] and its acceptable safety profile, prospective, double-blinded, randomised controlled trials to further assess its efficacy and safety in these patients are warranted [46]. One such randomised, double-blind controlled phase II/III trial is currently underway to investigate the efficacy of rituximab compared with cyclophosphamide in 116 patients with CTD, including scleroderma, over 48 weeks [57]. The primary outcome is change in FVC, with secondary outcomes including changes from baseline in the following parameters: DLCO, QoL, progression-free survival and global disease activity score [57]. The trial is due to complete in November 2020 [57].

Antifibrotic treatment is also being evaluated for use in SSc-ILD $[58,59]$. Preliminary findings from a case report study in five patients indicated that pirfenidone has shown improvements in FVC in patients with SSc-ILD [60]. Other treatment options include autologous haematopoietic stem cell transplantation, which has been designed to "reset" an auto-reactive immune system and ameliorate SSc [46]. Early success of a number of small phase I and II studies [61-63] has led to the conception of phase II/III multicentre trials comparing autologous haematopoietic stem cell transplantation to monthly cycles of intravenous cyclophosphamide in patients with SSc-ILD, suggesting that this may be a viable treatment option for this patient population $[46,64,65]$. Outcomes from the Autologous Stem Cell Transplantation International Scleroderma study, a randomised phase III trial comparing the efficacy and safety of haematopoietic stem cell transplantation (HSCT) and cyclophosphamide, showed a long-term survival benefit despite an early treatment-related mortality rate and increase in serious AEs $[66,67]$. Outcomes from a large phase III US-based trial comparing myeloablative autologous stem cell transplantation with cyclophosphamide treatment for severe scleroderma also demonstrated long-term benefits. Improved event-free and overall survival was observed in patients undergoing HSCT compared with patients receiving cyclophosphamide. However, toxicity was greater for the HSCT group versus the cyclophosphamide group [68].

\section{Hypersensitivity pneumonitis}

Chronic hypersensitivity pneumonitis can often be misdiagnosed as IPF [69]. Currently there are no approved therapies for hypersensitivity pneumonitis. Management involves identifying and eradicating the antigen from the patient's environment [70, 71]. Corticosteroids may be administered for acute symptomatic relief in those patients with subacute progressive and chronic disease [72]. While the management of acute/subacute hypersensitivity pneumonitis may be straightforward, chronic 
hypersensitivity pneumonitis does not always respond to corticosteroid treatment [72]. Recent studies have demonstrated some benefit with immunosuppressive therapy such as MMF or azathioprine with prednisone. These treatments have been associated with reduced decline in FVC \% pred, improved DLCO and transplant-free survival and a reduction in treatment-related AEs. Prospective randomised studies are needed to validate their effectiveness in these patients and to fully elucidate their role in the management of this disease $[73,74]$.

\section{Sarcoidosis}

Systemic corticosteroids can be used to treat the symptoms of sarcoidosis or may be used empirically in asymptomatic disease. No firm guidelines exist on if, when and how treatment should be initiated, but the decision to treat is centred around three factors: risk of severe dysfunction or irreversible damage to major organs, risk of death or the presence of incapacitating, constitutional symptoms [75]. In patients with mild cough, inhaled corticosteroids may be beneficial, while in patients with parenchymal lung disease between 20 and $40 \mathrm{mg}$ of prednisone daily is recommended for approximately 3 months before tapering to a lower dose or maintenance therapy [76]. Those patients with persistent symptoms, or progressively worsening disease may be eligible for additional immunosuppressive therapy [76], such as leflunomide [77, 78], methotrexate [79] or MMF [80-82]. Each drug has demonstrated some effectiveness in patients with sarcoidosis; however, these treatments require further investigation in randomised, controlled studies. One phase II study of the TNF- $\alpha$ inhibitor infliximab in combination with stable background doses of corticosteroid and/or immunosuppressive therapy, demonstrated statistically significant improvements in lung function in patients with symptomatic pulmonary sarcoidosis [83]. However, as treatment benefit did not translate to end-points such as improved QoL, 6-min walking distance or Borg's CR10 dyspnoea score on the overall study population, the clinical relevance of this treatment remains unclear [83]. Thus, further investigations are needed to determine if this is an effective treatment for these patients. Despite the differences between IPF and fibrotic pulmonary sarcoidosis, the potential exists for antifibrotic agents that are used for the management of IPF to be evaluated in sarcoidosis [84].

\section{ILDs related to other occupational exposures}

Patients diagnosed with ILDs related to other occupational exposures are at risk of developing a progressive phenotype. These diseases include Coal miner's pneumoconiosis, asbestosis and silicosis, all of which are associated with repeated exposure to mineral dust in the work environment [85]. To date, no favourable interventions have been demonstrated to reverse fibrotic disease progression. Therefore, management is limited to advising ILD patients to cease working in the environment in which they are exposed to the mineral dust causing progressive fibrosis [86].

\section{The importance of safe and effective treatments}

A significant proportion of patients with fibrosing ILDs that may present a progressive phenotype do not receive therapy as physicians may consider patients to have either mild or slowly progressing disease or even end-stage lung disease, while some physicians do not feel that the currently available therapies are effective or well tolerated [59]. In some countries, there may be no reimbursement for treatment. Therefore, as there are no established treatment regimens for this patient population, there is an urgent unmet need for effective, well-tolerated therapies for these patients.

There are a number of clinical and mechanistic parallels between IPF and other fibrosing ILDs that may present a progressive phenotype [87-89]; for example, IPF and SSc-ILD have some overlapping pathogenic mechanisms, including lung parenchymal injury and transforming growth factor- $\beta$-mediated fibroblast activation and myofibroblast accumulation [87-89]. The fibrotic pathways in hypersensitivity pneumonitis are less well understood, although the fibroblast precursors trafficking to the injured lung and promoting fibrosis is a mechanism common to both IPF and hypersensitivity pneumonitis $[6,87]$. In addition, data collected for a number of different fibrosing ILDs that may present a progressive phenotype, other than IPF, indicate that it is the ILD injury pattern and severity of destruction to the lung architecture that have the greatest impact on patient outcomes. The prognosis and clinical behaviour of ILDs that may present a progressive-fibrosing phenotype, especially those with a usual interstitial pneumonia pattern are similar to those seen in IPF [87]. Also, in a significant proportion of patients with progressive-fibrosing ILDs, the natural history appears to follow a disease course similar to IPF (i.e. worsening symptoms, lung function and health-related QoL), with variable rates of progression.

The mechanistic and clinical parallels between these pulmonary conditions suggest that the currently available antifibrotic agents, nintedanib and pirfenidone, may be viable therapeutic options for patients with progressive fibrosing ILDs other than IPF [1]. In IPF, antifibrotic treatments are usually prescribed with the aim of slowing disease progression. In fibrosing ILDs with a progressive phenotype, PF-ILD, a number of studies are underway to assess if antifibrotic treatments could improve outcomes and slow 
TABLE 1 Randomised controlled trials evaluating antifibrotic treatments in patients with interstitial lung disease (ILD)

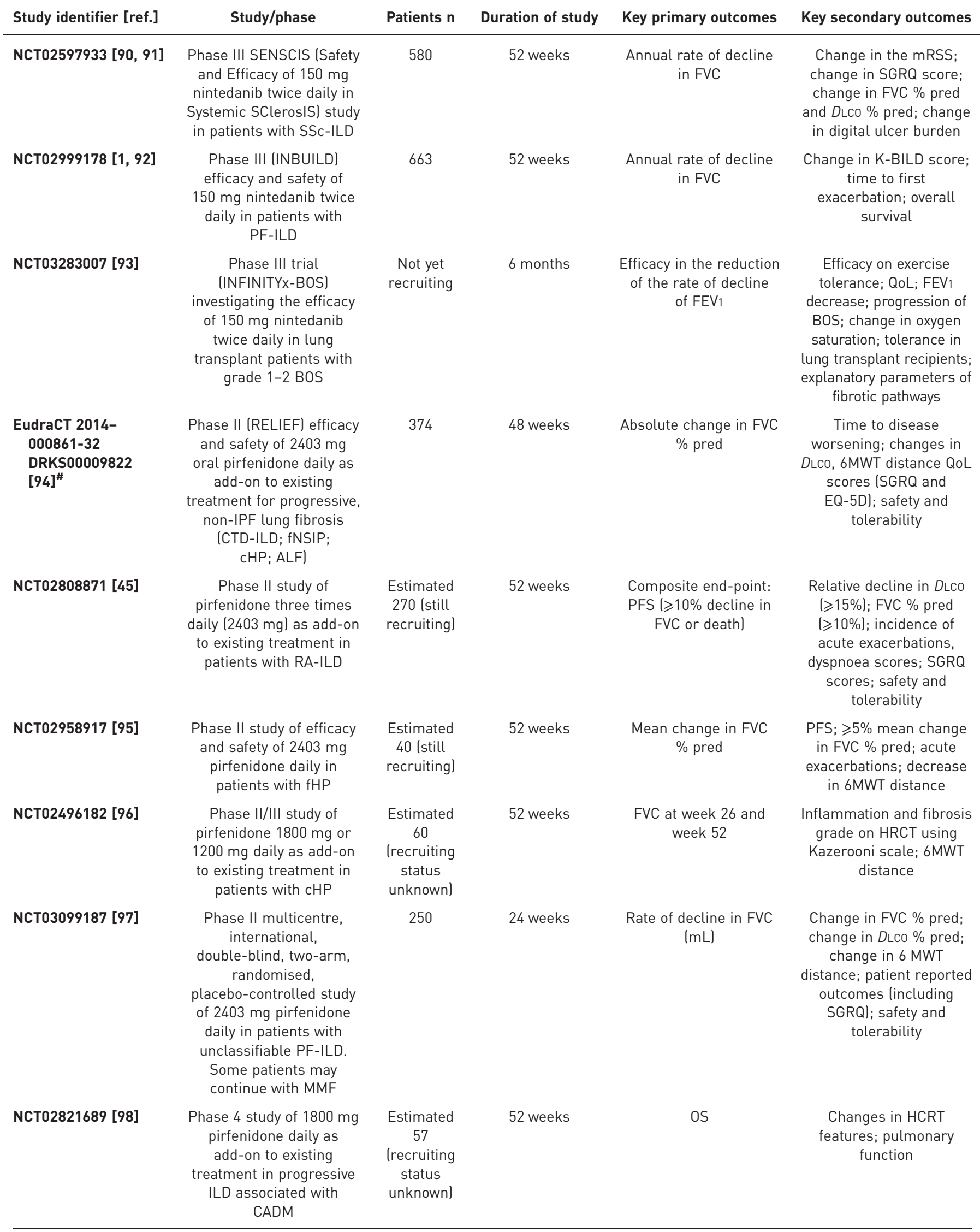


TABLE 1 Continued

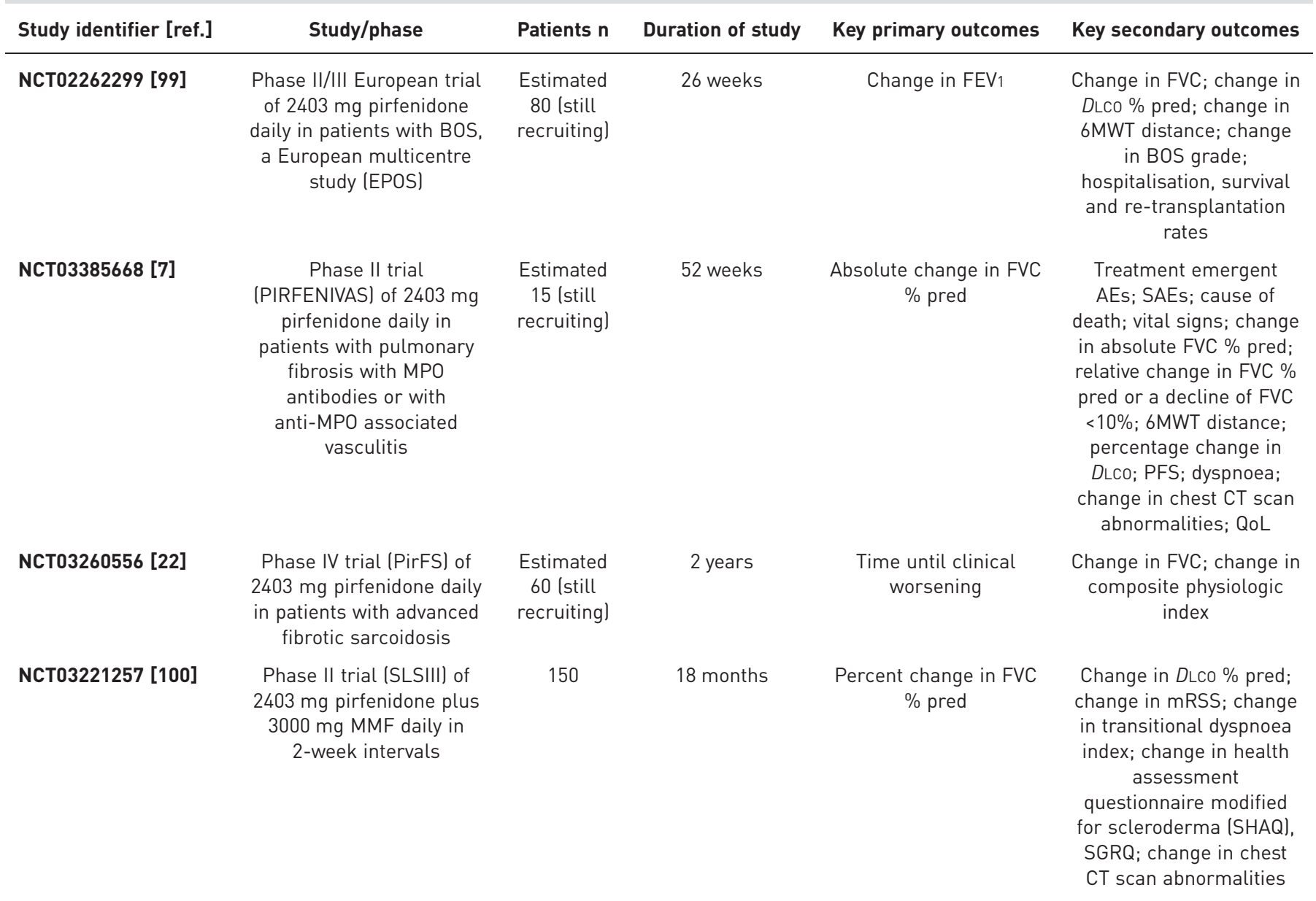

SSc-ILD: systemic sclerosis-associated ILD; FVC: forced vital capacity; mRSS: modified Rodnan skin score; SGRQ: St George's Respiratory Questionnaire; DLCO: diffusing capacity of the lung for carbon monoxide; PF-ILD: progressive-fibrosing ILD; K-BILD: King's Brief Interstitial Lung Disease questionnaire; BOS: bronchiolitis obliterans; FEV1: forced expiratory volume in $1 \mathrm{~s}$; QoL: quality of life; IPF: idiopathic pulmonary fibrosis; CTD-ILD: connective tissue disease associated-ILD; fNSIP: fibrotic nonspecific interstitial pneumonia; cHP: chronic hypersensitivity pneumonitis; ALF: asbestos-related lung fibrosis; EQ-5D: EuroQol 5-level questionnaire; RA-ILD: rheumatoid arthritis-associated ILD; PFS: progression-free survival; fHP: fibrotic hypersensitivity pneumonitis; 6MWT: 6-min walk test; HRCT: high-resolution computed tomography; MMF: mycophenolate mofetil; CADM: clinically amyopathic dermatomyositis; AE: adverse event; MPO: myeloperoxidase; SAE: serious adverse event; CT: computed tomography. " : prematurely ended. Reproduced and modified from [87] with permission.

down disease progression. However, it is important to note that these treatments are not intended to replace the use of drugs targeting the underlying condition, particularly the autoimmune components of CTD-ILDs. Observational and uncontrolled studies investigating nintedanib or pirfenidone in patients with these diseases have highlighted potential benefits, leading to initiation of a number of randomised controlled trials to further investigate these therapeutic agents (table 1) [87].

\section{Nintedanib for patients with ILDs}

The mode of action of nintedanib is well established, with outcomes from various studies demonstrating its consistent antifibrotic and anti-inflammatory activity in various animal models of lung fibrosis, thus providing a strong rationale for its clinical efficacy in patients with IPF and, therefore, patients with other progressive-fibrosing ILDs [17, 101].

Nintedanib for patients with fibrosing ILDs that may present a progressive phenotype other than IPF A phase III, prospective, multicentre randomised, double-blind, placebo-controlled study (INBUILD; ClinicalTrials.gov identifier: NCT02999178) has been designed to investigate the efficacy and safety of nintedanib in patients with fibrosing ILDs that may present a progressive phenotype other than IPF over 52 weeks [1]. This study aims to randomise (1:1) 600 patients with PF-ILD other than IPF to receive 
nintedanib twice daily or placebo. The blinded trial will end once the final randomised patient reaches week 52, and the benefit-risk profile of nintedanib during the study period has been assessed. Should the benefit-risk be positive then all patients will be given the option to receive open-label nintedanib [1]. As with the INPULSIS studies, the primary end-point is the annual rate of decline in FVC over 52 weeks. The absolute change from baseline in the King's Brief Interstitial Lung Disease questionnaire total score, time to first acute ILD exacerbation or death, and time to death will also be assessed over 52 weeks [1]. The estimated study completion date is August 2019 [92].

\section{Nintedanib for patients with SSc-ILD}

To date, nintedanib has been shown to effectively inhibit the endogenous and cytokine-induced activation of SSc fibroblasts with potent antifibrotic effects in various complementary mouse models [102]. A phase III, randomised, double-blind, placebo-controlled study evaluating the efficacy and safety of nintedanib in patients with SSc-ILD over 52 weeks (maximum 100 weeks) (SENSCIS; ClinicalTrials.gov identifier: NCT02597933) is currently ongoing [90]. An estimated 520 patients with a diagnosis of SSc (for $<7$ years before screening; $<5$ years prior to a protocol amendment in January 2017) or ILD ( $\geqslant 10 \%$ fibrosis of the lungs on central review) with an FVC $\geqslant 40 \%$ pred and DLCO $30-89 \%$ of predicted value receiving stable

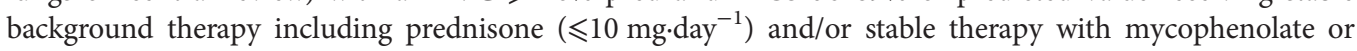
methotrexate ( $\geqslant 6$ months) at the time of study entry will be randomised 1:1 to receive nintedanib $150 \mathrm{mg}$ twice daily or placebo [90]. The primary end-point is the annual rate of decline in FVC over the study period, while key secondary end-points include absolute change from baseline in the modified Rodnan Skin Score and St George's Respiratory Questionnaire score at 52 weeks. The study will end when the last patient has completed 52 weeks of treatment and a follow-up visit 28 days later [91].

\section{Pirfenidone for patients with ILDs}

While the mode of action of pirfenidone is not yet completely known, its antifibrotic and anti-inflammatory activities have been well established in a number of IPF clinical trials, thus, making it another potential candidate for the management of fibrosing ILDs that may present a progressive phenotype other than IPF $[15,27,94]$.

Pirfenidone for patients with fibrosing ILDs that may present a progressive phenotype other than IPF A phase II, prospective, randomised, placebo-controlled study (RELIEF) had been designed to evaluate the efficacy and safety of pirfenidone in patients with fibrosing ILDs that may present a progressive phenotype other than IPF, including collagen-vascular disease-associated lung fibrosis, fibrotic iNSIP, chronic hypersensitivity pneumonitis and asbestos-related lung fibrosis [94]. The key efficacy end-point was to be the absolute change in FVC \% pred from baseline to week 48, while key secondary end-points include time to disease worsening, defined as time to clinical deterioration, lung fibrosis-related death, lung transplant or respiratory hospitalisation, whichever comes first [94]. This study has prematurely ended and no results are anticipated.

\section{Pirfenidone for patients with SSC-ILD}

The open-label, safety and tolerability LOTUSS trial is evaluating pirfenidone use in patients with SSc-ILD. A total of 63 patients were randomised to receive pirfenidone (conventional up-titration from $801 \mathrm{mg} \cdot \mathrm{day}^{-1}$ to $2403 \mathrm{mg} \cdot \mathrm{day}^{-1}$ ) over 2 or 4 weeks [87]. Pirfenidone was associated with an acceptable tolerability profile, and despite clinical outcomes not being the primary objective of this phase II study, both the per cent predicted FVC and the DLCO values remained unchanged for the study duration $[58,87]$. The phase III, multicentre, double-blind, parallel-group, randomised and placebo-controlled Scleroderma Lung Study III is evaluating the use of pirfenidone (titrated up to a target dose of $801 \mathrm{mg}$ taken three times daily) in combination with mycophenolate mofetil (MMF), (titrated up to a target dose of $1500 \mathrm{mg}$ taken twice daily) every 2 weeks for 18 months in 150 patients with SSc-ILD [100]. The results of this trial will determine whether the antifibrotic effects of pirfenidone will complement the delayed anti-inflammatory and immunosuppressive effects of MMF, to produce a significantly more rapid and/or greater improvement in lung function over time.

Pirfenidone for patients with RA, SSc-ILD, hypersensitivity pneumonitis and unclassifiable fibrosing ILD A number of phase II trials are also planned or ongoing to assess the efficacy and safety of pirfenidone either alone or as add-on treatment (table 1). Outcomes from these trials will provide valuable data to help define the role of this antifibrotic agent in the treatment of these fibrosing ILDs that may present a progressive phenotype other than IPF [87]. 


\section{Conclusions}

ILD encompasses a group of many different pulmonary conditions, of which IPF is the classic prototypic progressive fibrosing form. Clinical data suggest that a significant proportion of patients with other ILDs may develop an IPF-like progressive fibrosing phenotype. The current treatment regimens for these patients involve off-label use of corticosteroids and/or immunosuppressive therapies; however, these treatments are often associated with AEs leading to treatment discontinuation. In addition, these drugs have an unknown efficacy in patients with ILDs other than IPF; therefore, targeted antifibrotic therapies might be a valuable alternative. While not intended to replace the use of drugs targeting the underlying condition, antifibrotic therapies have a promising potential in fibrosing ILDs with a progressive phenotype (other than IPF), in improving outcomes and slowing disease progression. Both nintedanib and pirfenidone have demonstrated effective antifibrotic and anti-inflammatory effects in vitro and slowed disease progression in patients with IPF and are currently being evaluated as therapeutic treatment options for patients with other fibrosing ILDs that may present a progressive phenotype in the hope of providing a safe and effective treatment for this large group of patients.

Acknowledgements: The authors meet criteria for authorship as recommended by the International Committee of Medical Journal Editors. Writing assistance was provided by Tanya Chaudry of GeoMed, an Ashfield company, part of UDG Healthcare plc, which was contracted and funded by Boehringer Ingelheim Pharmaceuticals, Inc. (BIPI). BIPI was given the opportunity to review the manuscript for medical and scientific accuracy as well as intellectual property considerations.

Conflict of interest: L. Richeldi reports grants and personal fees from InterMune, personal fees from Sanofi-Aventis, Roche, ImmuneWorks, Shionogi, Boehringer Ingelheim, Celgene, Nitto, Fibrogen, Promedior, Bristol Myers Squibb and DynaMed, outside the submitted work. F. Varone reports personal fees from Boehringer Ingelheim and Hoffmann-La Roche, outside the submitted work. M. Bergna received research grants from Novartis, GSK, Boehringer Ingelheim, Roche, AstraZeneca and Sanofi, and advisory and investigator fees from GSK, AstraZeneca and Novartis. J. de Andrade reports grants from NIH/NHLBI, grants and personal fees from Boehringer Ingelheim and Genentech, and grants from Fibrogen and GBT, outside the submitted work. J. Falk has nothing to disclose. R. Hallowell has nothing to disclose. S. Jouneau reports grants from AIRB, Boehringer Ingelheim, LVL, Novartis and Roche, and personal fees from Actelion, AIRB, AstraZeneca, BMS, Boehringer Ingelheim, Chiesi, GSK, LVL, Mundipharma, Novartis, Pfizer and Roche, outside the submitted work. Y. Kondoh reports personal lecture fees from Boehringer Ingelheim Co. which is relevant to the presentation work. Y. Kondoh also reports advisory board fees and personal fees from Asahi Kasei Pharma Corp., Ltd, and personal fees from Eisai Inc., Kyorin Pharmaceutical Co., Ltd, Novartis Pharma K.K., Shionogi \& Co., Ltd and Teijin Pharma Ltd outside the submitted work. L. Morrow reports grants from Boehringer Ingelheim and Genentech, outside the submitted work. W. Randerath reports nonfinancial support from Boehringer, during the conduct of the study; and grants from Boehringer Ingelheim and Roche Pharma, outside the submitted work. M. Strek reports grants from Boehringer and Roche; and personal fees from Boehringer Ingelheim, outside the submitted work. G. Tabaj has nothing to disclose.

Support statement: The authors received no direct compensation related to the development of the manuscript. Writing assistance was contracted and funded by Boehringer Ingelheim Pharmaceuticals Inc. (BIPI).

\section{References}

1 Flaherty KR, Brown KK, Wells AU, et al. Design of the PF-ILD trial: a double-blind, randomised, placebo-controlled phase III trial of nintedanib in patients with progressive fibrosing interstitial lung disease. BMJ Open Respir Res 2017; 4: e000212.

2 Mikolasch TA, Porter JC. Transbronchial cryobiopsy in the diagnosis of interstitial lung disease: a cool new approach. Respirology 2014; 19: 623-624.

3 Richeldi L, Collard HR, Jones MG. Idiopathic pulmonary fibrosis. Lancet 2017; 389: 1941-1952.

4 Cottinn V, Hirani NA, Hotchkin DL, et al. Presentation, diagnosis and clinical course of the spectrum of progressive fibrosing interstitial lung diseases. Eur Respir Rev 2017; 26: 180076.

5 Travis WD, Costabel U, Hansell DM, et al. An official American Thoracic Society/European Respiratory Society statement: update of the international multidisciplinary classification of the idiopathic interstitial pneumonias. Am J Respir Crit Care Med 2013; 188: 733-748.

6 Raghu G, Rochwerg B, Zhang Y, et al. An Official ATS/ERS/JRS/ALAT clinical practice guideline: treatment of idiopathic pulmonary fibrosis. An update of the 2011 clinical practice guideline. Am J Respir Crit Care Med 2015; 192: e3-19.

7 London J, Ait el Ghaz S. Pilot study of pirfenidone in pulmonary fibrosis with anti-myeloperoxydase antibodies (PIRFENIVAS). https://clinicaltrials.gov/ct2/show/NCT03385668 Date last updated: March 19, 2018. Date last accessed: May 21, 2018.

8 European Medicines Agency. Esbriet ${ }^{\star}$ Summary of Product Characteristics. www.medicines.org.uk/emc/product/ 2731/smpc Date last updated: April 27, 2018. Date last accessed: May 23, 2018.

9 Richeldi L, Cottin V, du Bois RM, et al. Nintedanib in patients with idiopathic pulmonary fibrosis: combined evidence from the TOMORROW and INPULSIS((R)) trials. Respir Med 2016; 113: 74-79.

10 Raghu G, Collard HR, Egan JJ, et al. An official ATS/ERS/JRS/ALAT statement: idiopathic pulmonary fibrosis: evidence-based guidelines for diagnosis and management. Am J Respir Crit Care Med 2011; 183: 788-824.

11 Raghu G, Richeldi L. Current approaches to the management of idiopathic pulmonary fibrosis. Respir Med 2017; 129: $24-30$

12 Scullion T, Gorman P, Gibson E, et al. Antifibrotic therapy in idiopathic pulmonary fibrosis. Ulster Med J 2017; 86: 73. 
Richeldi L, Costabel U, Selman M, et al. Efficacy of a tyrosine kinase inhibitor in idiopathic pulmonary fibrosis. N Engl J Med 2011; 365: 1079-1087.

14 Richeldi L, du Bois RM, Raghu G, et al. Efficacy and safety of nintedanib in idiopathic pulmonary fibrosis. N Engl J Med 2014; 370: 2071-2082.

15 King TE Jr, Bradford WZ, Castro-Bernardini S, et al. A phase 3 trial of pirfenidone in patients with idiopathic pulmonary fibrosis. N Engl J Med 2014; 370: 2083-2092.

16 Hilberg F, Roth GJ, Krssak M, et al. BIBF 1120: triple angiokinase inhibitor with sustained receptor blockade and good antitumor efficacy. Cancer Res 2008; 68: 4774-4782.

17 Wollin L, Wex E, Pautsch A, et al. Mode of action of nintedanib in the treatment of idiopathic pulmonary fibrosis. Eur Respir J 2015; 45: 1434-1445.

18 Roth GJ, Binder R, Colbatzky F, et al. Nintedanib: from discovery to the clinic. J Med Chem 2015; 58: 1053-1063.

19 Wollin L, Ostermann A, Williams C. Nintedanib inhibits pro-fibrotic mediators from T cells with relevance to connective tissue disease-associated interstitial lung disease. Eur Respir J 2017; 50: Suppl. 61, PA903.

20 Varone F, Sgalla G, Iovene B, et al. Nintedanib for the treatment of idiopathic pulmonary fibrosis. Expert Opin Pharmacother 2018; 19: 167-175.

21 European Medicines Agency. Science Medicines Health. OFEV ${ }^{\circledast}$ (nintedanib). https://www.ema.europa.eu/en/ medicines/human/EPAR/ofev Date last updated: Sept 9, 2017. Date last accessed: May 21, 2018.

22 Baughman RP, Reeves R. Pirfenidone for Progressive Fibrotic Sarcoidosis (PirFS). https://clinicaltrials.gov/ct2/ show/NCT03260556 Date last updated: Sept 28, 2017. Date last accessed: May 21, 2018.

23 Macias-Barragan J, Sandoval-Rodriguez A, Navarro-Partida J, et al. The multifaceted role of pirfenidone and its novel targets. Fibrogenesis Tissue Repair 2010; 3: 16.

24 Wells AU, Brown KK, Flaherty KR, et al. What's in a name? That which we call IPF, by any other name would act the same. Eur Respir J 2018; 51: 1800692.

25 Roche. Esbriet ${ }^{\oplus}$ US prescribing information. www.gene.com/download/pdf/esbriet_prescribing.pdf Date last updated: Oct 1, 2017. Date last accessed: May 21, 2018.

26 European Medicines Agency. Science Medicines Health. Esbriet ${ }^{\oplus}$ (pirfenidone) authorisation details. https://www. ema.europa.eu/en/medicines/human/EPAR/esbriet Date last updated: May 7, 2018. Date last accessed: May 21, 2018 .

27 Noble PW, Albera C, Bradford WZ, et al. Pirfenidone in patients with idiopathic pulmonary fibrosis (CAPACITY): two randomised trials. Lancet 2011; 377: 1760-1769.

28 Shionogi \& Co., Ltd. Pharmaceuticals and Medical Devices Agency, Japan. Pirespa (pirfenidone) authorisation details. www.pmda.go.jp/files/000153687.pdf. Date last updated: Sept 16, 2008. Date last accessed: May 21, 2018.

29 Kinder BW, Shariat C, Collard HR, et al. Undifferentiated connective tissue disease-associated interstitial lung disease: changes in lung function. Lung 2010; 188: 143-149.

30 Nunes H, Schubel K, Piver D, et al. Nonspecific interstitial pneumonia: survival is influenced by the underlying cause. Eur Respir J 2015; 45: 746-755.

31 Travis WD, Hunninghake G, King TE Jr, et al. Idiopathic nonspecific interstitial pneumonia: report of an American Thoracic Society project. Am J Respir Crit Care Med 2008; 177: 1338-1347.

32 Yamakawa H, Kitamura H, Takemura T, et al. Prognostic factors and disease behaviour of pathologically proven fibrotic non-specific interstitial pneumonia. Respirology 2018; 23: 1032-1040.

33 Kondoh Y, Taniguchi H, Yokoi T, et al. Cyclophosphamide and low-dose prednisolone in idiopathic pulmonary fibrosis and fibrosing nonspecific interstitial pneumonia. Eur Respir J 2005; 25: 528-533.

34 Tomassetti S, Ryu JH, Piciucchi S, et al. Nonspecific interstitial pneumonia: what is the optimal approach to management? Semin Respir Crit Care Med 2016; 37: 378-394.

35 Lee SH, Park MS, Kim SY, et al. Factors affecting treatment outcome in patients with idiopathic nonspecific interstitial pneumonia: a nationwide cohort study. Respir Res 2017; 18: 204.

36 Corte TJ, Ellis R, Renzoni EA, et al. Use of intravenous cyclophosphamide in known or suspected, advanced non-specific interstitial pneumonia. Sarcoidosis Vasc Diffuse Lung Dis 2009; 26: 132-138.

37 Ryerson CJ, Urbania TH, Richeldi L, et al. Prevalence and prognosis of unclassifiable interstitial lung disease. Eur Respir J 2013; 42: 750-757.

38 Nakamura Y, Sugino K, Kitani M, et al. Clinico-radio-pathological characteristics of unclassifiable idiopathic interstitial pneumonias. Respir Investig 2018; 56: 40-47.

39 American Thoracic Society, European Respiratory Society. American Thoracic Society/European Respiratory Society International Multidisciplinary Consensus Classification of the Idiopathic Interstitial Pneumonias. Am J Respir Crit Care Med 2002; 165: 277-304.

40 Bluett J, Jani M, Symmons DPM. Practical management of respiratory comorbidities in patients with rheumatoid arthritis. Rheumatol Ther 2017; 4: 309-332.

41 Iqbal K, Kelly C. Treatment of rheumatoid arthritis-associated interstitial lung disease: a perspective review. Ther Adv Musculoskelet Dis 2015; 7: 247-267.

42 Shaw M, Collins BF, Ho LA, et al. Rheumatoid arthritis-associated lung disease. Eur Respir Rev 2015; 24 : 1-16.

43 Md Yusof MY, Kabia A, Darby M, et al. Effect of rituximab on the progression of rheumatoid arthritis-related interstitial lung disease: 10 years' experience at a single centre. Rheumatology (Oxford) 2017; 56: 1348-1357.

44 Fernandez-Diaz C, Loricera J, Castaneda S, et al. Abatacept in patients with rheumatoid arthritis and interstitial lung disease: a national multicenter study of 63 patients. Semin Arthritis Rheum 2018; 48: 22-27.

45 Rosas I. Phase 11 study of pirfenidone in patients with RAILD. https:/clinicaltrials.gov/ct2/show/NCT02808871 Date last updated: June 14, 2017. Date last accessed: May 21, 2018.

46 Silver KC, Silver RM. Management of systemic-sclerosis-associated interstitial lung disease. Rheum Dis Clin North Am 2015; 41: 439-457.

47 Tashkin DP, Elashoff R, Clements PJ, et al. Cyclophosphamide versus placebo in scleroderma lung disease. N Engl J Med 2006; 354: 2655-2666.

48 Tashkin DP, Elashoff R, Clements PJ, et al. Effects of 1-year treatment with cyclophosphamide on outcomes at 2 years in scleroderma lung disease. Am J Respir Crit Care Med 2007; 176: 1026-1034.

49 Domiciano DS, Bonfa E, Borges CT, et al. A long-term prospective randomized controlled study of non-specific interstitial pneumonia (NSIP) treatment in scleroderma. Clin Rheumatol 2011; 30: 223-229. 
Fischer A, Brown KK, Du Bois RM, et al. Mycophenolate mofetil improves lung function in connective tissue disease-associated interstitial lung disease. J Rheumatol 2013; 40: 640-646.

Tzouvelekis A, Galanopoulos N, Bouros E, et al. Effect and safety of mycophenolate mofetil or sodium in systemic sclerosis-associated interstitial lung disease: a meta-analysis. Pulm Med 2012; 2012: 143637.

Tashkin DP, Roth MD, Clements PJ, et al. Mycophenolate mofetil versus oral cyclophosphamide in scleroderma-related interstitial lung disease (SLS II): a randomised controlled, double-blind, parallel group trial. Lancet Respir Med 2016; 4: 708-719.

Daoussis D, Liossis SN, Tsamandas AC, et al. Experience with rituximab in scleroderma: results from a 1-year, proof-of-principle study. Rheumatology (Oxford) 2010; 49: 271-280.

Jordan S, Distler JH, Maurer B, et al. Effects and safety of rituximab in systemic sclerosis: an analysis from the European Scleroderma Trial and Research (EUSTAR) group. Ann Rheum Dis 2015; 74: 1188-1194.

Sharp C, McCabe M, Dodds N, et al. Rituximab in autoimmune connective tissue disease-associated interstitial lung disease. Rheumatology (Oxford) 2016; 55: 1318-1324.

Daoussis D, Liossis SN, Tsamandas AC, et al. Effect of long-term treatment with rituximab on pulmonary function and skin fibrosis in patients with diffuse systemic sclerosis. Clin Exp Rheumatol 2012; 30: S17-S22.

Maher T. Rituximab versus cyclophosphamide in connective tissue disease-ILD (RECITAL). https://clinicaltrials. gov/ct2/show/NCT01862926 Date last updated: June 8, 2017. Date last accessed: May 21, 2018.

Khanna D, Albera C, Fischer A, et al. An open-label, phase II study of the safety and tolerability of pirfenidone in patients with scleroderma-associated interstitial lung disease: the LOTUSS trial. J Rheumatol 2016; 43: $1672-1679$.

Kreuter M, Olson A, Fischer A, et al. Current treatment of patients with non-IPF progressive fibrosing interstitial lung disease. Am J Respir Crit Care Med 2018; 197: A4273.

Miura Y, Saito T, Fujita K, et al. Clinical experience with pirfenidone in five patients with scleroderma-related interstitial lung disease. Sarcoidosis Vasc Diffuse Lung Dis 2014; 31: 235-238.

Binks M, Passweg JR, Furst D, et al. Phase I/II trial of autologous stem cell transplantation in systemic sclerosis: procedure related mortality and impact on skin disease. Ann Rheum Dis 2001; 60: 577-584.

Farge D, Passweg J, van Laar JM, et al. Autologous stem cell transplantation in the treatment of systemic sclerosis: report from the EBMT/EULAR Registry. Ann Rheum Dis 2004; 63: 974-981.

Nash RA, McSweeney PA, Crofford LJ, et al. High-dose immunosuppressive therapy and autologous hematopoietic cell transplantation for severe systemic sclerosis: long-term follow-up of the US multicenter pilot study. Blood 2007; 110: 1388-1396.

Burt RK, Shah SJ, Dill K, et al. Autologous non-myeloablative haemopoietic stem-cell transplantation compared with pulse cyclophosphamide once per month for systemic sclerosis (ASSIST): an open-label, randomised phase 2 trial. Lancet 2011; 378: 498-506.

van Laar JM, Naraghi K, Tyndall A. Haematopoietic stem cell transplantation for poor-prognosis systemic sclerosis. Rheumatology (Oxford) 2015; 54: 2126-2133.

Suzuki A, Kondoh Y, Fischer A. Recent advances in connective tissue disease related interstitial lung disease. Expert Rev Respir Med 2017; 11: 591-603.

van Laar JM, Farge D, Sont JK, et al. Autologous hematopoietic stem cell transplantation $v$ s intravenous pulse cyclophosphamide in diffuse cutaneous systemic sclerosis: a randomized clinical trial. JAMA 2014; 311: 2490-2498.

Sullivan KM, Goldmuntz EA, Keyes-Elstein L, et al. Myeloablative autologous stem-cell transplantation for severe scleroderma. N Engl J Med 2018; 378: 35-47.

Andrade J, Schwarz M, Collard HR, et al. The Idiopathic Pulmonary Fibrosis Clinical Research Network (IPFnet): diagnostic and adjudication processes. Chest 2015; 148: 1034-1042.

Fernandez Perez ER, Swigris JJ, Forssen AV, et al. Identifying an inciting antigen is associated with improved survival in patients with chronic hypersensitivity pneumonitis. Chest 2013; 144: 1644-1651.

Raghu G. Interstitial lung disease. In: Goldman L, Schafer AL, eds. Goldman-Cecil Medicine, 25th Edn. Philadelphia, Elsevier, 2016.

Miyazaki Y, Tsutsui T, Inase N. Treatment and monitoring of hypersensitivity pneumonitis. Expert Rev Clin Immunol 2016; 12: 953-962.

Adegunsoye A, Oldham JM, Fernandez Perez ER, et al. Outcomes of immunosuppressive therapy in chronic hypersensitivity pneumonitis. ERJ Open Res 2017; 3: 00016-02017.

Morisset J, Johannson KA, Vittinghoff E, et al. Use of mycophenolate mofetil or azathioprine for the management of chronic hypersensitivity pneumonitis. Chest 2017; 151: 619-625.

Valeyre D, Prasse A, Nunes H, et al. Sarcoidosis. Lancet 2014; 383: 1155-1167.

Rheumatol 2018; 30: 243-248. 43-48.

Sahoo DH, Bandyopadhyay D, Xu M, et al. Effectiveness and safety of leflunomide for pulmonary and extrapulmonary sarcoidosis. Eur Respir J 2011; 38: 1145-1150.

Korsten P, Strohmayer K, Baughman RP, et al. Refractory pulmonary sarcoidosis - proposal of a definition and recommendations for the diagnostic and therapeutic approach. Clin Pulm Med 2016; 23: 67-75.

Vorselaars ADM, Wuyts WA, Vorselaars VMM, et al. Methotrexate $v s$ azathioprine in second-line therapy of sarcoidosis. Chest 2013; 144: 805-812.

Brill AK, Ott SR, Geiser T. Effect and safety of mycophenolate mofetil in chronic pulmonary sarcoidosis: a retrospective study. Respiration 2013; 86: 376-383.

Hamzeh N, Voelker A, Forssen A, et al. Efficacy of mycophenolate mofetil in sarcoidosis. Respir Med 2014; 108: 1663-1669.

Baughman RP, Drent M, Kavuru M, et al. Infliximab therapy in patients with chronic sarcoidosis and pulmonary involvement. Am J Respir Crit Care Med 2006; 174: 795-802.

Patterson KC, Strek ME. Pulmonary fibrosis in sarcoidosis. Clinical features and outcomes. Ann Am Thorac Soc 2013; 10: 362-370. 
Beer C, Kolstad HA, Sondergaard K, et al. A systematic review of occupational exposure to coal dust and the risk of interstitial lung diseases. Eur Clin Respir J 2017; 4: 1264711.

Perret JL, Plush B, Lachapelle P, et al. Coal mine dust lung disease in the modern era. Respirology 2017; 22: 662-670.

Kreuter M, Walscher J, Behr J. Antifibrotic drugs as treatment of nonidiopathic pulmonary fibrosis interstitial pneumonias: the time is now (?). Curr Opin Pulm Med 2017; 23: 418-425.

Solomon JJ, Olson AL, Fischer A, et al. Scleroderma lung disease. Eur Respir Rev 2013; 22: 6-19. idiopathic pulmonary fibrosis: how similar and distinct? Arthritis Rheumatol 2014; 66: 1967-1978.

A trial to compare nintedanib with placebo for patients with scleroderma-related lung fibrosis. https:// clinicaltrials.gov/ct2/show/NCT02597933 Date last updated: May 17, 2018. Date last accessed: May 21, 2018.

Distler O, Brown KK, Distler JHW, et al. Design of a randomised, placebo-controlled clinical trial of nintedanib in patients with systemic sclerosis-associated interstitial lung disease (SENSCIS). Clin Exp Rheumatol 2017; 35: Suppl. 106, 75-81.

Efficacy and safety of nintedanib in patients with progressive fibrosing interstitial lung disease (PF-ILD). https:// clinicaltrials.gov/ct2/show/NCT02999178 Date last updated: May 4, 2018. Date last accessed: July 1, 2018.

Brugière $O$. Nintedanib in lung transplant recipients with bronchiolitis obliterans syndrome grade 1-2 (INFINITx-BOS). https://clinicaltrials.gov/ct2/show/NCT03283007 Date last updated: April 27, 2018. Date last accessed: May 21, 2018.

Behr J, Neuser P, Prasse A, et al. Exploring efficacy and safety of oral pirfenidone for progressive, non-IPF lung fibrosis (RELIEF) - a randomized, double-blind, placebo-controlled, parallel group, multi-center, phase II trial. BMC Pulm Med 2017; 17: 122

Fier K. Study of efficacy and safety of pirfenidone in patients with fibrotic hypersensitivity pneumonitis. https:// clinicaltrials.gov/ct2/show/NCT02958917 Date last updated: May 3, 2017. Date last accessed: May 21, 2018.

Pena P. Pirfenidone in the chronic hypersensitivity pneumonitis treatment (Picheon). https:/clinicaltrials.gov/ct2/ show/NCT02496182 Date last updated: July 14, 2015. Date last accessed: May 21, 2018.

A study of pirfenidone in patients with unclassifiable progressive fibrosing interstitial lung disease. https:// clinicaltrials.gov/ct2/show/NCT03099187 Date last updated: April 9, 2018. Date last accessed: May 21, 2018.

Ye S. Pirfenidone in progressive interstitial lung disease associated with clinically amyopathic dermatomyositis. https://clinicaltrials.gov/ct2/show/NCT02821689 Date last updated: July 1, 2016. Date last accessed: May 21, 2018.

Perch M. European trial of pirfenidone in BOS, a European multi-center study (EPOS). https://clinicaltrials.gov/ ct2/show/NCT02262299 Date last updated: December 12, 2016. Date last accessed: May 21, 2018.

Roth M. Scleroderma Lung Study III - Combining Pirfenidone With Mycophenolate (SLSIII). https:// clinicaltrials.gov/ct2/show/NCT03221257 Date last updated: Sept 10, 2018. Date last accessed: Oct 24, 2018.

Wollin L, Maillet I, Quesniaux V, et al. Antifibrotic and anti-inflammatory activity of the tyrosine kinase inhibitor nintedanib in experimental models of lung fibrosis. J Pharmacol Exp Ther 2014; 349: 209-220.

Huang J, Beyer C, Palumbo-Zerr K, et al. Nintedanib inhibits fibroblast activation and ameliorates fibrosis in preclinical models of systemic sclerosis. Ann Rheum Dis 2016; 75: 883-890. 